\title{
Comparison of Bootstrap Confidence Intervals for Impulse Responses of German Monetary Systems ${ }^{1}$
}

\author{
Alexander Benkwitz, Helmut Lütkepohl \\ Humboldt-Universität \\ Institut für Statistik und Ökonometrie \\ Spandauer Straße 1 \\ D - 10178 Berlin, Germany \\ and \\ Jürgen Wolters \\ Freie Universität \\ Institut für Statistik und Ökonometrie \\ Boltzmannstraße 20 \\ D - 14195 Berlin, Germany
}

Key Words and Phrases: impulse response, bootstrap, money demand system, monetary policy

JEL Classification: C32

\begin{abstract}
It is argued that standard impulse response analysis based on vector autoregressive models has a number of shortcomings. Although the impulse responses are estimated quantities, measures for sampling variability such as confidence intervals are often not provided. If confidence intervals are given they are often based on bootstrap methods with poor theoretical properties. These problems are illustrated using two German monetary systems. Proposals are made for improving current practice. Special emphasis is placed on systems with cointegrated variables.

\footnotetext{
${ }^{1}$ We thank Michael H. Neumann for helpful comments, the Deutsche Forschungsgemeinschaft, SFB 373, and the European Commission under the Training and Mobility of Researchers Programme (contract No. ERBFMRXCT980213) for financial support.
} 


\section{Introduction}

Impulse responses are standard tools in vector autoregressive (VAR) analyses. In this context an economic system of interest is described by a VAR model which is estimated from the available time series data in unrestricted form or with various types of structural and statistical restrictions imposed. There are a number of problems related to commonly applied procedures. First of all, impulse responses are computed from estimated coefficients and are therefore also estimates. This fact is ignored in part of the literature where the point estimates are plotted only and the relation of the variables involved is interpreted on the basis of these point estimates without properly taking into account the estimation variability. In another part of the literature the estimation uncertainty of impulse responses is assessed by setting up confidence intervals (CIs). In many studies it was found, however, that the CIs are rather wide and, hence, the impulse responses are not very informative so that nothing much can be said about the actual underlying relations. Clearly this reflects the substantial sampling variability in the estimated VAR parameters which in turn is a consequence of estimating these quantities in a largely unrestricted model with many parameters.

Another potential problem in this context is that the CIs for the impulse responses are often based on bootstrap methods. It has been argued by Benkwitz, Lütkepohl \& Neumann (1998) (henceforth BLN) that the usual bootstrap procedure used in this context can fail completely by producing CIs with actual coverage probability of zero, regardless of the desired nominal confidence level. In other words, the bootstrap CIs may give a grossly distorted impression of the range of likely impulse responses for a VAR model.

The purpose of this article is to illustrate and discuss the importance of these problems for applied work. We will use two small German monetary systems and show that it is crucial to take into account the estimation uncertainty when interpreting impulse responses in the context of dynamic econometric models. We argue that the commonly used bootstrap methods for determining CIs of impulse responses are problematic and we point out that the method favored by Hall (1992) is advantageous. Moreover we show that imposing restrictions on the short-term dynamics of a system can result in major improvements in the precision of the estimated impulse responses which in turn can lead to a more informative picture of the dynamic interactions between the variables of the system under consideration. In our analysis we will focus on vector error correction models (VECMs) and we will pay special 
attention to the treatment of cointegration relations.

The paper has the following structure. The general framework of the analysis is presented in the next section and inference on impulse responses is considered in Section 3. In particular, alternative methods for computing bootstrap CIs for impulse responses are discussed. These methods are applied and compared within two small monetary systems for Germany in Section 4. Conclusions are drawn in Section 5.

The following notation is used throughout: $\mathcal{L}(X)$ denotes the distribution of the random variable $X$. The natural logarithm is abbreviated as $\log$ and $\Delta$ is the differencing operator defined such that for a time series variable $y_{t}, \Delta y_{t}=y_{t}-y_{t-1}$. Nonstationary variables which become stationary upon differencing once are referred to as $I(1)$ variables.

\section{Analysis of VAR Processes}

Many macroeconomic analyses are based on linear dynamic models of the type

$$
A_{0} y_{t}=A_{1} y_{t-1}+\cdots+A_{p} y_{t-p}+\Psi x_{t}+\Xi D_{t}+u_{t}
$$

where $y_{t}=\left[y_{1 t}, \ldots, y_{K t}\right]^{\prime}$ is a $K$-dimensional vector of observable endogenous variables, the $A_{i}(i=0,1, \ldots, p)$ are $(K \times K)$ coefficient matrices, $x_{t}$ represents a vector of $N$ unmodelled observable variables, $D_{t}$ contains all deterministic terms such as seasonal dummy variables, intercepts and polynomial trend terms, $\Psi$ and $\Xi$ are also coefficient matrices and $u_{t}=\left[u_{1 t}, \ldots, u_{K t}\right]^{\prime}$ is a white noise process, that is, the $u_{t}$ are serially uncorrelated or independent with zero mean and nonsingular (positive definite) covariance matrix $\Sigma_{u}$. The model (2.1) is somewhat more general than the typical pure VAR model in that it may contain unmodelled variables whereas in standard VAR analyses all stochastic variables are treated as endogenous. We will still refer to (2.1) as our basic VAR model. The maximum lag length $p$ of the endogenous variables is usually referred to as the order of the VAR process and the process is briefly called a $\operatorname{VAR}(p)$. The process may be stationary or it may contain $I(1)$ variables and $r$ cointegrating relations, where $0<r<K$. In the latter case it is often written as a VECM,

$$
\Gamma_{0} \Delta y_{t}=\alpha \beta^{\prime} y_{t-1}+\Gamma_{1} \Delta y_{t-1}+\cdots+\Gamma_{p-1} \Delta y_{t-p+1}+\Psi x_{t}+\Xi D_{t}+u_{t}
$$

where the $\Gamma_{j}(j=0,1, \ldots, p-1)$ are the short-run parameter matrices, $\alpha$ is the $(K \times r)$ loading matrix and $\beta$ is a $(K \times r)$ matrix containing $r$ linearly independent cointegration 
relations. In the examples in Section 4 the exogenous variables $x_{t}$ are stationary variables.

Regardless of the stationarity properties, the model in (2.1) or (2.2) summarizes the instantaneous and intertemporal relations between the variables. The exact form of these relations is usually difficult to see directly from the coefficients, especially if there are only just identifying restrictions on the short-term parameters $\Gamma_{i}(i=0,1, \ldots, p-1)$. Therefore impulse response functions are often computed which represent the marginal responses of the endogenous variables of the system to an impulse in one of the endogenous variables. These may be regarded as conditional forecasts of the endogenous variables given that they have been zero up to time 0 when an impulse in one of the variables occurs. Depending on the kind of impulse hitting the system there are various different impulse responses that have been used for interpreting VAR models. For detailed discussions see Sims (1980, 1981), Lütkepohl (1990, 1991), Watson (1994), Lütkepohl \& Breitung (1997). The important property of these quantities from the point of view of our analysis is that they are particular nonlinear functions of the parameters of the model in (2.1) or (2.2), say,

$$
\phi_{i j, h}=\phi_{i j, h}\left(A_{0}, A_{1}, \ldots, A_{p}\right)=\phi_{i j, h}\left(\alpha, \beta, \Gamma_{0}, \Gamma_{1}, \ldots, \Gamma_{p-1}\right)
$$

where $\phi_{i j, h}$ represents the response of variable $i$ to an impulse in variable $j, h$ periods ago. Precise formulas for different versions of impulse responses may be found in Lütkepohl (1991, Chapter 2) or Lütkepohl \& Breitung (1997), for instance. Because the VECM in (2.2) can always be written in the equivalent levels form in (2.1) and vice versa and because our example models in Section 4 are VECMs we will focus on the latter version in the following in order to minimize repetition. The VECM is also the more convenient model form for discussing the treatment of cointegration relations.

\section{Inference on Impulse Responses}

Usually the coefficients of the model in (2.2) are estimated by some standard procedure such as (pseudo) maximum likelihood (ML) or feasible generalized least squares (GLS) possibly estimating the cointegration parameters in a first stage and keeping them fixed in estimating the other parameters. Estimators of the impulse responses are then obtained as

$$
\hat{\phi}_{i j, h}=\phi_{i j, h}\left(\hat{\alpha}, \hat{\beta}, \hat{\Gamma}_{0}, \hat{\Gamma}_{1}, \ldots, \hat{\Gamma}_{p-1}\right)
$$


where the $\hat{\alpha}, \hat{\beta}, \hat{\Gamma}_{0}, \hat{\Gamma}_{1}, \ldots, \hat{\Gamma}_{p-1}$ are the estimated VECM parameter matrices. Under general assumptions the resulting impulse responses have asymptotic normal distributions which may be used for constructing CIs. In practice, bootstrap methods are often used for this purpose, because these methods occasionally lead to more reliable small sample inference than CIs based on standard asymptotic theory. However we want to emphasize the fact that both approaches, standard asymptotics and the bootstrap, are based on asymptotic arguments.

The analytical expressions of the asymptotic variances of the impulse response coefficients are rather complicated. Using the bootstrap for setting up CIs, the precise expressions of the variances are not needed and, hence, deriving the analytical expressions can be avoided. In the following we will discuss some methods that have been proposed in this context.

The following bootstrap method will be considered:

(1) Estimate the parameters of the model in (2.2) by a suitable procedure.

(2) Generate bootstrap residuals $u_{1}^{*}, \ldots, u_{T}^{*}$ by randomly drawing with replacement from the set of estimated and recentered residuals, $\left\{\hat{u}_{1}-\bar{u}_{.}, \ldots, \hat{u}_{T}-\bar{u}.\right\}$, where $\hat{u}_{t}=\hat{\Gamma}_{0} \Delta y_{t}-$ $\hat{\alpha} \hat{\beta}^{\prime} y_{t-1}-\hat{\Gamma}_{1} \Delta y_{t-1}-\cdots-\hat{\Gamma}_{p-1} \Delta y_{t-p+1}-\hat{\Psi} x_{t}-\hat{\Xi} D_{t}$, and $\bar{u}_{.}=T^{-1} \sum \hat{u}_{t}$.

(3) Set $\left(y_{-p+1}^{*}, \ldots, y_{0}^{*}\right)=\left(y_{-p+1}, \ldots, y_{0}\right)$ and construct bootstrap time series recursively using the levels representation given in (2.1),

$$
y_{t}^{*}=\hat{A}_{0}^{-1}\left(\hat{A}_{1} y_{t-1}^{*}+\cdots+\hat{A}_{p} y_{t-p}^{*}+\hat{\Psi} x_{t}+\hat{\Xi} D_{t}+u_{t}^{*}\right), \quad t=1, \ldots, T .
$$

(4) Reestimate the parameters $\Gamma_{0}, \Gamma_{1}, \ldots \Gamma_{p-1}, \Psi, \Xi, \alpha, \beta$ from the generated data.

(5) Calculate a bootstrap version of the statistic of interest, say $\hat{\phi}_{i j, h}^{*}$, based on the parameter estimates obtained in Stage (4).

In Stage (4) where the bootstrap estimates are computed there are two alternative ways to do so. The first possibility is to use the same estimation method in each bootstrap replication that was used in estimating the VECM coefficients from the original data. In this procedure the cointegration matrix $\beta$ is reestimated for each bootstrap sample. Alternatively one may argue that the $\beta$ matrix is estimated superconsistently from the original data and is therefore treated as known and fixed in the bootstrap replications. We will explore these two possibilities in the context of the examples in Section 4. 
In the following we use the symbols $\phi, \hat{\phi}_{T}$ and $\hat{\phi}_{T}^{*}$ to denote some general impulse response coefficient, its estimator implied by the estimators of the model coefficients and the corresponding bootstrap estimator, respectively. The subscript $T$ indicates the sample size.

The most commonly used method in setting up CIs for impulse responses in practice proceeds by using $\gamma / 2$ - and $(1-\gamma / 2)$-quantiles, say $s_{\gamma / 2}^{*}$ and $s_{(1-\gamma / 2)}^{*}$, respectively, of the bootstrap distribution $\mathcal{L}\left(\hat{\phi}_{T}^{*} \mid y_{-p+1}, \ldots, y_{0}, \ldots, y_{T} ; x_{1}, \ldots, x_{T}\right)$, and defining

$$
C I_{S}=\left[s_{\gamma / 2}^{*}, s_{(1-\gamma / 2)}^{*}\right]
$$

The interval $C I_{S}$ is the percentile confidence interval described, e.g., by Efron \& Tibshirani (1993). These authors point out, however, that it may not have the desired coverage probability. This problem occurs for example if $\hat{\phi}_{T}$ is a biased estimator of $\phi$. In that case the bootstrap distribution may be asymptotically centered at $\phi$ plus a bias term and, hence, $C I_{S}$ is a $(1-\gamma) 100 \%$ CI for the latter quantity and may have a grossly distorted level as a CI for $\phi$. To fix this drawback modifications of $C I_{S}$ were proposed in the literature. In the context of impulse response analysis Kilian (1998) has suggested a method to reduce the problem if the data generation process is a stationary VAR. Since in practice we often have to deal with processes containing integrated nonstationary variables we will not use this variant here, in particular, since our example models in Section 4 involve cointegrated variables. Moreover, Kilian's modification has not been used much in applications and its asymptotic properties are not fully clear. In fact, it is shown by BLN that the asymptotic coverage probability for a nominal $(1-\gamma) 100 \% C I_{S}$ can be zero for any $\gamma$ strictly between zero and one. Therefore we will present another bootstrap variant (see Hall (1992, Chapter 3)) which overcomes some of the problems of the standard interval.

Let $t_{\gamma / 2}^{*}$ and $t_{(1-\gamma / 2)}^{*}$ be the $\gamma / 2$ - and $(1-\gamma / 2)$-quantiles of

$$
\mathcal{L}\left(\hat{\phi}_{T}^{*}-\hat{\phi}_{T} \mid y_{-p+1}, \ldots, y_{0}, \ldots, y_{T} ; x_{1}, \ldots, x_{T}\right),
$$

respectively. According to the usual bootstrap analogy,

$$
\mathcal{L}\left(\hat{\phi}_{T}-\phi\right) \approx \mathcal{L}\left(\hat{\phi}_{T}^{*}-\hat{\phi}_{T} \mid y_{-p+1}, \ldots, y_{0}, \ldots, y_{T} ; x_{1}, \ldots, x_{T}\right)
$$

one gets the interval

$$
C I_{H}=\left[\hat{\phi}_{T}-t_{(1-\gamma / 2)}^{*}, \hat{\phi}_{T}-t_{\gamma / 2}^{*}\right]
$$


Hall (1992) calls this CI "percentile interval". Therefore, in the following we refer to the method leading to $C I_{H}$ as Hall's percentile method, whereas the method underlying $C I_{S}$ is referred to as the standard method. If $\mathcal{L}\left(\sqrt{T}\left(\hat{\phi}_{T}^{*}-\hat{\phi}_{T}\right) \mid y_{-p+1}, \ldots, y_{0}, \ldots, y_{T} ; x_{1}, \ldots, x_{T}\right)$ has the same limit distribution as $\mathcal{L}\left(\sqrt{T}\left(\hat{\phi}_{T}-\phi\right)\right)$ it follows immediately that $C I_{H}$ has the correct size asymptotically, that is, $\operatorname{Pr}\left(\phi \in C I_{H}\right) \longrightarrow 1-\gamma$ as $T \rightarrow \infty$ and, hence, Hall's percentile method is asymptotically correct.

It is well established in the bootstrap literature that the quality of the bootstrap approximation of the distribution of a general statistic $\hat{\mu}_{T}$, say, can be improved by reducing its dependence on the unknown distribution that governs the data generating process. For example, with respect to the sample mean of i.i.d. random variables it is well-known that studentizing leads to a better rate of approximation by the bootstrap (see, e.g., Hall (1992)). Therefore, it may be advantageous to use a studentized statistic $\left(\hat{\phi}_{T}-\phi\right) / \sqrt{\widehat{\operatorname{var}}\left(\hat{\phi}_{T}\right)}$ as a basis for constructing confidence intervals. Hence in the present context it may be advantageous to determine a bootstrap quantile based on the statistic $\left(\hat{\phi}_{T}^{*}-\hat{\phi}_{T}\right) / \sqrt{\widehat{\operatorname{var}}\left(\hat{\phi}_{T}^{*}\right)}$. In this approach the variances are also estimated by a bootstrap, that is,

$$
\widehat{\operatorname{var}}\left(\hat{\phi}_{T}\right)=\frac{1}{B^{*}-1} \sum_{i=1}^{B^{*}}\left(\hat{\phi}_{T}^{*, i}-\overline{\hat{\phi}_{T}^{*}}\right)^{2}
$$

and

$$
\widehat{\operatorname{var}}\left(\hat{\phi}_{T}^{*}\right)=\frac{1}{B^{* *}-1} \sum_{i=1}^{B^{* *}}\left(\hat{\phi}_{T}^{* *, i}-\overline{\hat{\phi}_{T}^{* *}}\right)^{2}
$$

where $\hat{\phi}_{T}^{* *, i}$ is obtained by a double bootstrap, that is, pseudo-data are generated according to a process obtained on the basis of the bootstrap systems parameters and $B^{*}$ and $B^{* *}$ are the respective numbers of bootstrap replications in the first and second stages (see Hall (1992) for details).

Let $t_{\gamma / 2}^{* *}$ and $t_{(1-\gamma / 2)}^{* *}$ be the $\gamma / 2$ - and $(1-\gamma / 2)$-quantiles, respectively, of

$$
\mathcal{L}\left(\left(\hat{\phi}_{T}^{*}-\hat{\phi}_{T}\right) / \sqrt{\widehat{\operatorname{var}}\left(\hat{\phi}_{T}^{*}\right)} \mid y_{-p+1}, \ldots, y_{0}, \ldots, y_{T} ; x_{1}, \ldots, x_{T}\right) .
$$

Using these quantiles we get the studentized Hall interval

$$
C I_{S H}=\left[\hat{\phi}_{T}-t_{(1-\gamma / 2)}^{* *} \sqrt{\widehat{\operatorname{var}}\left(\hat{\phi}_{T}\right)}, \hat{\phi}_{T}-t_{\gamma / 2}^{* *} \sqrt{\widehat{\operatorname{var}}\left(\hat{\phi}_{T}\right)}\right]
$$

which also has an asymptotically correct coverage probability if $(3.2)$ and $\mathcal{L}\left(\left(\hat{\phi}_{T}-\phi\right) / \sqrt{\widehat{\operatorname{var}}\left(\hat{\phi}_{T}\right)}\right)$ have identical proper limiting distributions. In the next section we will use these CIs in analyzing the impulse responses of two German monetary systems. 


\section{Analysis of German Monetary Systems}

Brüggemann \& Wolters (1998) (BW) and Lütkepohl \& Wolters (1998) (LW) consider small models for the German monetary sector to investigate the channels of monetary policy. LW use M3 as measure of the money stock whereas BW consider a system for the more narrow measure M1. In both studies impulse responses are used to analyze the dynamic interactions of the variables in VECMs which can be represented in the form (2.2). Both studies do not report measures of sampling variability for the impulse responses and they conclude that the impact of the Bundesbank policy on inflation may have been quite limited since prices do not react strongly to changes in the money stock and to changes in the interest rate. In the following we will reconsider these results by checking the significance of the effects observed in the aforementioned articles. Moreover, we will demonstrate the effects of using different methods for computing bootstrap CIs. We will begin with a system presented by BW and then turn to LW.

\subsection{M1 System}

BW construct quarterly models for the period 1962(1) - 1989(4) and the extended period 1962(1) - 1996(2) using seasonally unadjusted data. In the following we will concentrate on the model version for the extended period which includes German unification in 1990 and allows for international price movements influencing domestic prices. The following variables are included in the system: $m 1_{t}$ is the logarithm of (nominal) M1; $y_{t}$ is the logarithm of real GNP; $p_{t}$ is the logarithm of the GNP deflator, hence, $(m 1-p)_{t}$ is the logarithm of real M1 and $\Delta p_{t}=p_{t}-p_{t-1}$ is the quarterly inflation rate; $R_{t}$ is a long-term interest rate ('Umlaufsrendite'); $p m_{t}$ is an import price index which is treated as an unmodelled variable reflecting the openness of the German economy and capturing the effects of exchange rates. The precise data sources are provided in the Appendix. In addition there are a number of deterministic variables in the model such as seasonal dummies and a shift dummy $S 90 q 3_{t}$ which takes into account the level shifts in $m 1_{t}$ and $y_{t}$ due to the German unification. It is zero until 1990(2) and afterwards it has the value one.

BW found that there is one cointegration relation between the $I(1)$ variables $m 1_{t}, p_{t}, y_{t}$ and $R_{t}$. For the period from 1961(4) to 1996(2) they found the following long-run money 
demand relation (see BW, Equation (3.4))

$$
(m 1-p)_{t}=1.105 y_{t}-5.133 R_{t}+0.407 S 90 q 3_{t}+e c 1_{t}
$$

Here $e c 1_{t}$ stands for the deviations from the long-run relation. The estimated VECM of BW is given in Table 1 except for deterministic terms. The model is estimated by Zellner's seemingly unrelated regressions method. Note that the model may be viewed as a reduced form because $\Gamma_{0}$ is an identity matrix. Moreover, the instantaneous residual correlation is quite small and therefore no orthogonalization is needed for computing meaningful impulse responses. This model is the result of a specification procedure described in detail in BW which initially also allows for instantaneous relations of the variables (i.e., $\Gamma_{0} \neq I$ ).

Since the model is in reduced form a fully unrestricted version with full rank error correction term may be estimated by considering the VAR form in (2.1) with order $p=5$. We have used that model to compute impulse responses together with all three versions of $95 \%$ bootstrap CIs $\left(C I_{S}, C I_{H}, C I_{S H}\right)$. The results based on 2000 bootstrap replications are plotted in Figure $1 .^{1}$ For $C I_{S H}$ we used 50 bootstrap drawings for estimating $\widehat{\operatorname{var}}\left(\hat{\phi}_{T}^{*}\right)$. Clearly in this case the differences between the methods are not substantial. Because in most cases the CIs are almost symmetric around the estimated impulse response coefficients it is not surprising that $C I_{S}$ and $C I_{H}$ are similar. Exceptions are, for instance, the response of $p$ to an impulse in the same variable and the response of $R$ to an impulse in $m 1$. Also, the $C I_{S H}$ intervals are in most cases quite similar to $C I_{H}$. An analogous result was also obtained for other cases considered in the following. Therefore we focus on $C I_{H}$ because it has the better theoretical basis than $C I_{S}$ (Hall (1992, pp. 128)) and it is much less computer intensive than $C I_{S H}$.

A major problem with the intervals in Figure 1 is that they are rather wide and, hence, the actual responses in the underlying system are quite uncertain if the CIs properly reflect the estimation variability. For example, based on the CIs in Figure 1, an impulse in $m 1$ does not have a significant effect on the price level. Moreover, an increase in the price level does not have a significant impact on income. Thus, an impulse response analysis based on the full unrestricted reduced form model does not give a clear indication of the relations

\footnotetext{
${ }^{1}$ The computations were performed with a GAUSS program. We have checked the sensitivity with respect to the number of bootstrap replications and found that very similar results are obtained if at least 1000 bootstrap replications are used.
} 
between the variables. The results in the figure also show the importance of computing CIs for the impulse responses because an interpretation that ignores the substantial estimation uncertainty may be quite misleading.

An improvement in the estimation precision can be expected from taking into account the restrictions imposed by BW. In Figure 2 the impulse responses and corresponding $C I_{H}$ intervals are shown which are obtained for the restricted VECM. The $C I_{H}$ intervals from the unrestricted VAR model are given for comparison purposes. Obviously, taking into account the restrictions results in a substantial improvement in the precision as expected. Now the response of $m 1$ to an impulse in the price level $p$ has become significant and the same holds for the response of $p$ to an impulse in $m 1$, for instance. Thus, the present analysis sheds doubt on the previous interpretation from BW that the impact of changes in $m 1$ on the price level may not be very strong.

Interestingly, in Figure 2 it can be seen that the impulse responses from the model with restrictions are in most cases within the CIs from the unrestricted model. On the other hand, the CIs from the restricted model do not always contain the estimates of the impulse responses from the unrestricted model. Hence, estimating the impulse responses from an unrestricted model does not only increase the uncertainty in the estimates but may also lead to quite different point estimates. There is more overlap between the CIs if intervals are computed from the restricted VECM and a VECM where only the cointegration restriction is imposed. These CIs are shown in Figure 3, where it is seen that the CIs from the less restricted model are substantially wider than the CIs from the restricted model. The longrun development of the impulse responses from both models is similar due to enforcing the cointegration restriction. It may also be worth noting that using the bootstrap for an unrestricted model may result in singularities in the asymptotic distributions of the estimated impulse responses. This in turn may lead to strongly distorted and, hence, unreliable bootstrap CIs as pointed out by BLN. Thus, using a restricted model is also useful for removing one source of problems for the bootstrap CIs.

The question whether to fix the estimated cointegration relation in the bootstrap or to reestimate it in each replication is addressed in Figure 4. In most cases there is nearly no difference in the CIs. If there are differences the CIs based on reestimated cointegration vectors tend to be larger. Of course, without a detailed analysis it is difficult to interpret this 
result because the reduced length intervals obtained by fixing the cointegration parameters may be the outcome of ignoring the estimation variability in the cointegration vector. Hence, it may cover up the actual estimation uncertainty that remains in the estimates. Without further knowledge on the properties of the estimates it may be preferable to reestimate the cointegration parameters in each bootstrap replication.

\subsection{M3 System}

Using seasonally unadjusted data for the period 1976(1) - 1996(4), LW construct a quarterly model for M3. They include similar variables as BW in their model. In addition to the variables defined in the context of the M1 model they use the following variables: $m 3_{t}$ is the logarithm of (nominal) M3 and, hence, $(m 3-p)_{t}$ is the logarithm of real M3; $(R-r)_{t}$ is the difference between the long-term interest rate and the own rate of M3, denoted by $r_{t}$, so that this variable represents the opportunity costs of holding M3 rather than longer term bonds, $d_{t}(R-r)_{t}$ is identical to $(R-r)_{t}$ for the period 1994(3) - 1995(4) and is zero otherwise, it is used to model a nonlinearity in the impact of the interest rate differential on the demand for money in the period mentioned. The variable is treated as a member of the group of unmodelled variables in (2.2). Again there are some additional deterministic variables such as seasonal dummies and dummies to take care of the unification.

LW find that the variables $(m 3-p)_{t}, y_{t}$ and $\Delta p_{t}$ are $I(1)$ and that there is one cointegration relation between these variables of the form (see LW, Equation (3.2))

$$
(m 3-p)_{t}=y_{t}-13.50 \Delta p_{t}+0.14 S 90 q 3_{t}+e c 3_{t}
$$

which may be interpreted as an essential part of a long-run money demand relation. Here $e c 3_{t}$ represents the deviations from the long-run relation. The estimated VECM of LW is given in Table 2 where deterministic terms are excluded as in Table 1 . The estimation method used is iterated three-stage least squares. The details of the specification procedure are provided by LW. Notice that the instantaneous $\Delta^{2} p_{t}$ appears in the $\Delta(m 3-p)_{t}$ equation and, hence, the model is a structural form in the sense that $\Gamma_{0}$ is not the identity matrix if the model is written in the form (2.2). It may also be worth noting that the instantaneous residual correlation is quite small so that interpreting the residuals as impulses to specific variables is justified. 
Rewriting the model in such a way that it looks like (2.1) or (2.2), an impulse response analysis can be carried out as described in Section 2. Since we now consider a model in structural form we compare again $C I_{S}$ and $C I_{H}$ to check whether a similar result is obtained as in the reduced form case. The impulse responses together with approximate $95 \%$ CIs are depicted in Figure 5 where the cointegration parameters are reestimated in each bootstrap replication. The impulse responses are identical to those in Figure 1 of LW. They still look a bit different because they have been scaled in a different way. The scaling in our Figure 5 is adjusted to the width of the CIs. Thus, it is less arbitrary than the scaling used by LW. It is seen in the figure that the two types of CIs are again very similar. The small differences indicate that some of the underlying distributions may not be symmetric. Moreover, Figure 5 reveals that impulses in money and the interest rate differential may have significant effects on the inflation rate. In other words, the Bundesbank's policy may have been more effective than suggested by Figure 1 of LW. Thereby the importance of providing measures for the estimation uncertainty of the impulse responses as in the figure is apparent.

\section{Conclusions}

In this study we have illustrated some problems related to standard impulse response analysis in VAR models and we have suggested alternative procedures. It has been demonstrated on the basis of two small monetary systems for Germany that it is very important to take into account that the commonly considered impulse responses are estimates and, hence, subject to some uncertainty. This estimation uncertainty has to be taken into account in the interpretation of the impulse responses. Plotting CIs together with point estimates of the impulse responses can provide a good picture of the uncertainty involved. In practice, in this context CIs are often based on bootstrap methods. We have argued that standard bootstrap CIs may be heavily distorted and therefore may be misleading. A simple alternative is proposed and applied for analyzing the two German monetary example systems. It is shown that the common practice of performing an impulse response analysis on the basis of a largely unrestricted model may not be very informative with respect to the actual relation of the variables because the estimation uncertainty can be substantial. Imposing restrictions on the parameters of the model can lead to substantial improvements in this respect. 
It should be noted, however, that there are a number of open questions regarding the properties of the procedures used in this study. First, the asymptotic and small sample properties of bootstrap CIs in the present context are not fully clear, especially if the model contains cointegrated variables. Although there is a range of Monte Carlo studies exploring the small sample properties of estimated impulse responses, most of these studies focus on stationary VAR processes. Moreover, the underlying data generation processes are necessarily quite limited compared to the wide range of models that have been used in applied work. Hence, it is not clear whether the simulation results are generalisable to a particular model under consideration in empirical work. Second, as is common in the empirical literature, we have constructed CIs for the individual impulse response coefficients. It may be more plausible from a conceptual point of view to consider joint confidence regions for the impulse response functions because not only individual impulse response coefficients but the overall shape of some response is often of interest.

In conclusion, it is clear that there are a number of open problems surrounding impulse response analysis in the context of VAR models. Despite these problems is is important to use the available tools for getting an impression of the uncertainty underlying any specific analysis. Therefore it is surprising that some popular software packages for dynamic econometric analysis do not provide confidence intervals for impulse responses and thereby complicate the interpretation of the results.

\section{Appendix. Data Sources}

Seasonally unadjusted quarterly data were used for the following variables taken from the given sources. All data refer to West Germany until 1990(2) and to the unified Germany afterwards.

M1: nominal monthly values from Monatsberichte der Deutschen Bundesbank; the quarterly values are the values of the last month of each quarter. The variable $m 1$ is log M1.

M3: nominal monthly values from Monatsberichte der Deutschen Bundesbank; the quarterly values are the values of the last month of each quarter. The variable $m 3$ is log M3.

GNP: quarterly real gross national product from Deutsches Institut für Wirtschaftsforschung, Volkswirtschaftliche Gesamtrechnung. The variable $y$ is log GNP. 
Price index: GNP deflator $(1991=100)$ from Deutsches Institut für Wirtschaftsforschung, Volkswirtschaftliche Gesamtrechnung. The variable $p$ is the logarithm of the price index.

Average bond rate (Umlaufsrendite) $(R)$ : monthly values from Monatsberichte der Deutschen Bundesbank; the quarterly value is the value of the last month of each quarter.

Own rate of M3 $(r)$ : the series was constructed from the interest rates of savings deposits $(r s)$ and the interest rates of 3-months time deposits $(r t)$ from Monatsberichte der Deutschen Bundesbank as a weighted average as follows:

$$
r= \begin{cases}0.24 r t+0.42 r s & \text { for } 1976(1)-1990(2) \\ 0.30 r t+0.33 r s & \text { for } 1990(3)-1996(4)\end{cases}
$$

The weights are chosen according to the relative shares of the corresponding components of M3. The quarterly value is the value of the last month of each quarter.

Import price index: PM $(1991=100)$ from Deutsches Institut für Wirtschaftsforschung, Volkswirtschaftliche Gesamtrechnung. The variable pm is the logarithm of PM.

The data may be obtained from the internet,

$$
\text { http://wotan.wiwi.hu-berlin.de/oekonometrie/engl/data.html }
$$

\section{References}

Benkwitz, A., H. Lütkepohl \& M. H. Neumann (1998). Problems related to confidence intervals for impulse responses of autoregressive processes. Econometric Reviews, forthcoming.

Brüggemann, I. \& J. Wolters (1998). Money and Prices in Germany: Empirical Results for 1962 to 1996. In: Galata, R. and Küchenhoff, H. (eds.), Econometrics in Theory and Practice, Heidelberg: Physica.

Efron, B. \& R. J. Tibshirani (1993). An Introduction to the Bootstrap, New York: Chapman \& Hall.

Hall, P. (1992). The Bootstrap and Edgeworth Expansion, New York: Springer. 
Kilian, L. (1998). Small-sample confidence intervals for impulse response functions. Review of Economics and Statistics 80, 218-230.

Lütkepohl, H. (1990). Asymptotic distributions of impulse response functions and forecast error variance decompositions of vector autoregressive models, Review of Economics and Statistics 72, 116-125.

Lütkepohl, H. (1991). Introduction to Multiple Time Series Analysis, Berlin: SpringerVerlag.

Lütkepohl, H. \& J. Breitung (1997). Impulse response analysis of vector autoregressive processes, in: C. Heij, J.M. Schumacher, B. Hanzon and C. Praagman (eds.), System Dynamics in Economic and Financial Models, Chichester: John Wiley, 299-326.

Lütkepohl, H. \& J. Wolters (1998). A money demand system for German M3, Empirical Economics 23, 371-386.

Sims, C. A. (1980). Macroeconomics and reality, Econometrica 48, 1-48.

Sims, C. A. (1981). An autoregressive index model for the U.S. 1948-1975, in: J. Kmenta \& J.B. Ramsey (eds.), Large-Scale Macro-Econometric Models, Amsterdam: NorthHolland, 283-327.

Watson, M. W. (1994). Vector autoregressions and cointegration, in: Engle, R.F. and D.L. McFadden (eds.), Handbook of Econometrics, Vol. IV, New York: Elsevier. 
Table 1: M1 System Without Deterministic Terms, Estimation Period 1962(1) - 1996(2)

\begin{tabular}{|c|c|c|c|c|}
\hline & $\Delta m 1_{t}$ & $\Delta p_{t}$ & $\Delta y_{t}$ & $\Delta R_{t}$ \\
\hline$e c 1_{t-1}$ & $\begin{array}{c}-0.115 \\
(6.6)^{a}\end{array}$ & & & \\
\hline$\Delta m 1_{t-1}$ & & & & $\begin{array}{c}0.036 \\
(2.1)\end{array}$ \\
\hline$\Delta m 1_{t-2}$ & & & $\begin{array}{c}0.191 \\
(3.0)\end{array}$ & \\
\hline$\Delta m 1_{t-3}$ & & & $\begin{array}{c}0.117 \\
(2.0)\end{array}$ & \\
\hline$\Delta p_{t-1}$ & & $\begin{array}{c}-0.133 \\
(2.6)\end{array}$ & & \\
\hline$\Delta p_{t-2}$ & & $\begin{array}{c}0.204 \\
(3.6)\end{array}$ & $\begin{array}{c}-0.284 \\
(3.0)\end{array}$ & \\
\hline$\Delta p_{t-4}$ & $\begin{array}{c}0.411 \\
(5.4)\end{array}$ & $\begin{array}{l}0.538 \\
(10.0)\end{array}$ & & \\
\hline$\Delta y_{t-2}$ & $\begin{array}{c}-0.231 \\
(4.9)\end{array}$ & $\begin{array}{c}0.082 \\
(3.7)\end{array}$ & $\begin{array}{c}-0.431 \\
(6.4)\end{array}$ & \\
\hline$\Delta y_{t-3}$ & & $\begin{array}{c}0.113 \\
(5.3)\end{array}$ & & \\
\hline$\Delta y_{t-4}$ & & & $\begin{array}{c}0.457 \\
(7.6)\end{array}$ & \\
\hline$\Delta R_{t-1}$ & $\begin{array}{c}-0.884 \\
(3.7)\end{array}$ & $\begin{array}{c}0.240 \\
(2.4)\end{array}$ & & $\begin{array}{c}0.192 \\
(2.3)\end{array}$ \\
\hline$\Delta p m_{t-4}$ & & $\begin{array}{c}0.058 \\
(2.4)\end{array}$ & & \\
\hline
\end{tabular}

${ }^{a}$ Absolute values of $t$-ratios in parentheses. 
Table 2: M3 System Without Deterministic Terms,

Estimation Period 1976(1) - 1996(4)

\begin{tabular}{|c|c|c|c|c|}
\hline & $\Delta(m 3-p)_{t}$ & $\Delta^{2} p_{t}$ & $\Delta y_{t}$ & $(R-r)_{t}$ \\
\hline$e c 3_{t-1}$ & $\begin{array}{l}-0.111 \\
(7.1)^{a}\end{array}$ & & $\begin{array}{c}0.044 \\
(2.3)\end{array}$ & \\
\hline$\Delta(m 3-p)_{t-1}$ & & $\begin{array}{c}-0.058 \\
(2.2)\end{array}$ & $\begin{array}{c}0.269 \\
(3.2)\end{array}$ & \\
\hline$\Delta(m 3-p)_{t-2}$ & & & $\begin{array}{c}0.172 \\
(2.5)\end{array}$ & \\
\hline$\Delta(m 3-p)_{t-3}$ & & $\begin{array}{c}0.089 \\
(2.8)\end{array}$ & & \\
\hline$\Delta(m 3-p)_{t-4}$ & $\begin{array}{c}-0.069 \\
(2.0)\end{array}$ & & & \\
\hline$\Delta^{2} p_{t}$ & $\begin{array}{c}-1.262 \\
(6.6)\end{array}$ & & & \\
\hline$\Delta^{2} p_{t-1}$ & & $\begin{array}{c}-1.086 \\
(11.3)\end{array}$ & & \\
\hline$\Delta^{2} p_{t-2}$ & & $\begin{array}{c}-1.044 \\
(9.5)\end{array}$ & & \\
\hline$\Delta^{2} p_{t-3}$ & & $\begin{array}{c}-0.747 \\
(6.9)\end{array}$ & & \\
\hline$\Delta^{2} p_{t-4}$ & $\begin{array}{c}-0.251 \\
(2.8)\end{array}$ & $\begin{array}{c}-0.258 \\
(2.7)\end{array}$ & & \\
\hline$\Delta y_{t-1}$ & $\begin{array}{c}-0.220 \\
(5.7)\end{array}$ & & $\begin{array}{c}-0.323 \\
(3.7)\end{array}$ & \\
\hline$\Delta y_{t-3}$ & & $\begin{array}{c}0.075 \\
(2.2)\end{array}$ & & \\
\hline$\Delta y_{t-4}$ & & $\begin{array}{c}0.096 \\
(3.6)\end{array}$ & $\begin{array}{c}0.243 \\
(3.4)\end{array}$ & \\
\hline$(R-r)_{t-1}$ & $\begin{array}{c}-0.568 \\
(4.9)\end{array}$ & & & $\begin{array}{l}0.836 \\
(12.2)\end{array}$ \\
\hline$(R-r)_{t-3}$ & $\begin{array}{l}0.427 \\
(2.8)\end{array}$ & & & \\
\hline$(R-r)_{t-4}$ & $\begin{array}{c}-0.406 \\
(2.8)\end{array}$ & & & $\begin{array}{c}-0.200 \\
(2.9)\end{array}$ \\
\hline$\Delta p m_{t-4}$ & & $\begin{array}{c}0.065 \\
(2.6)\end{array}$ & & $\begin{array}{c}0.055 \\
(2.2)\end{array}$ \\
\hline$d_{t}(R-r)_{t}$ & $\begin{array}{c}-0.430 \\
(7.6)\end{array}$ & & & \\
\hline
\end{tabular}

${ }^{a}$ Absolute values of $t$-ratios in parentheses. 

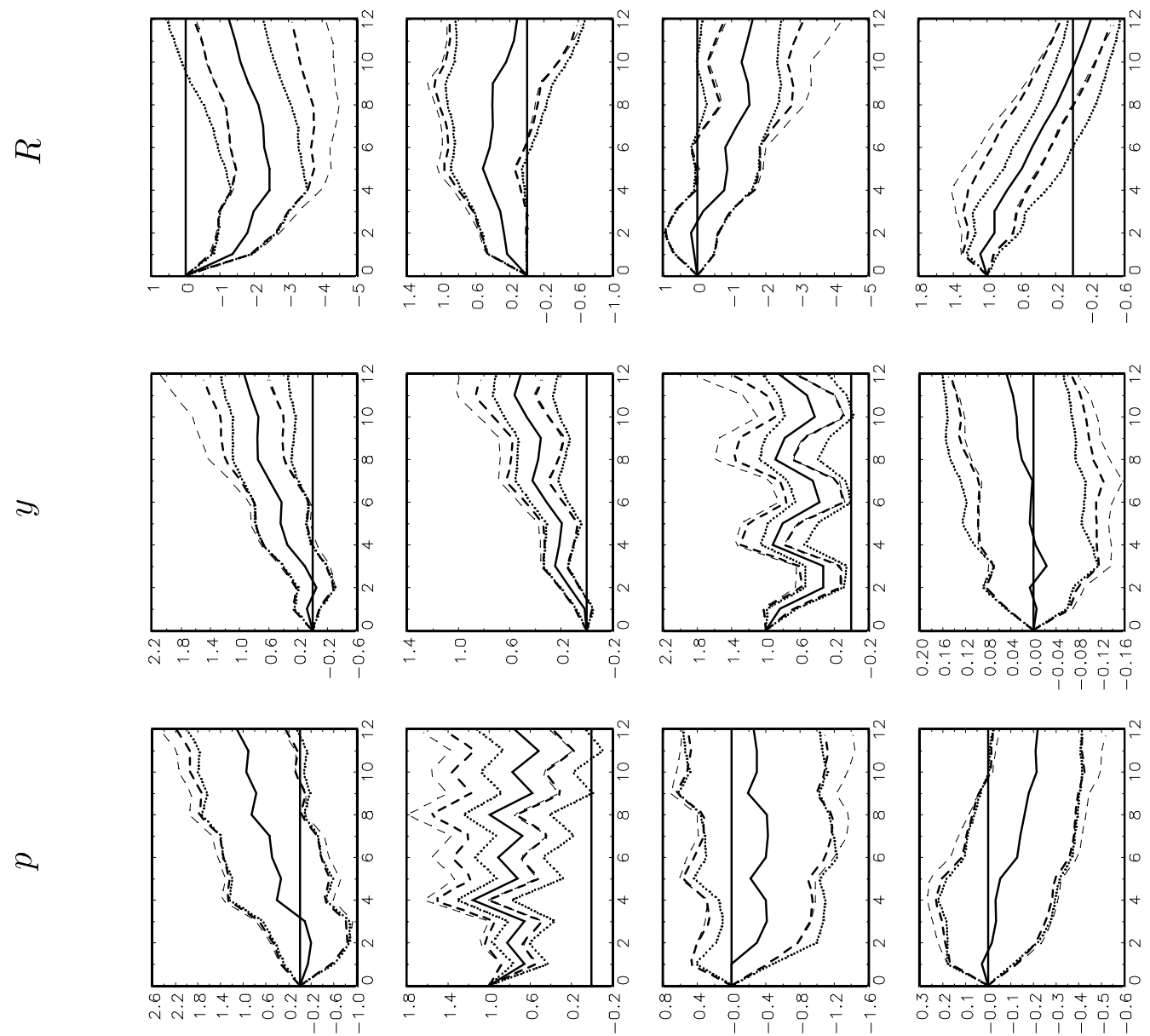

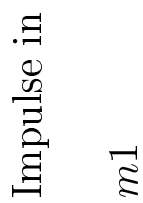
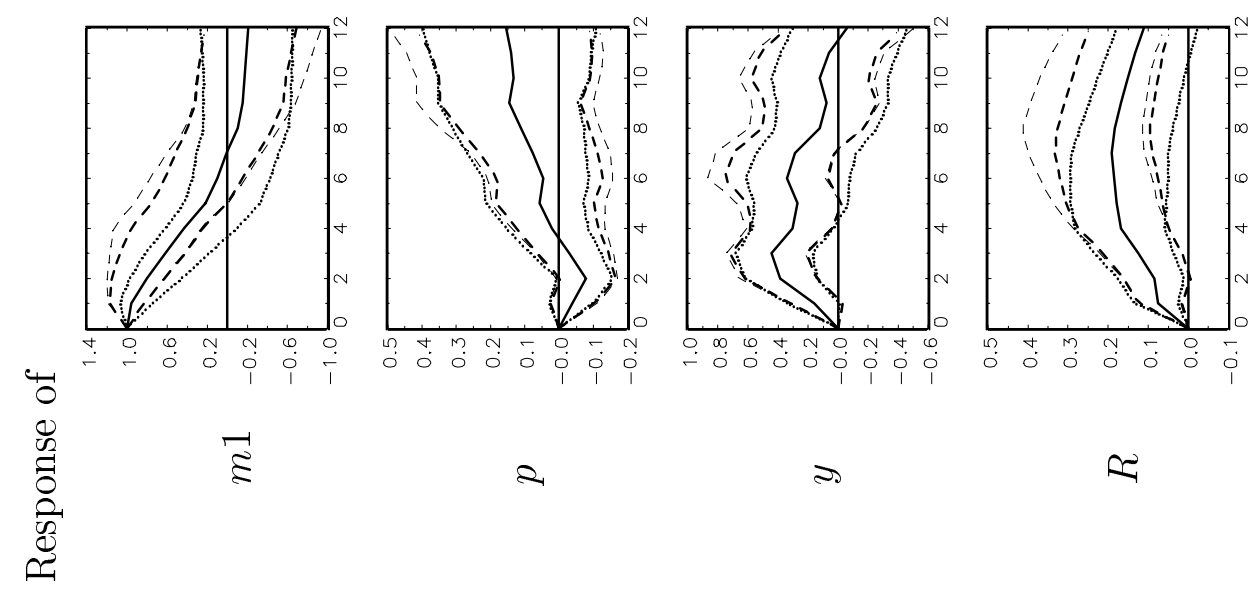

2

จ

$\sim$

Figure 1: Estimated impulse responses for fully unrestricted M1 VAR system (solid line) with 95\% CIs: $C I_{S}$ (dotted lines), $C I_{H}$ (strong dashed lines), $C I_{S H}$ (light dashed lines) 

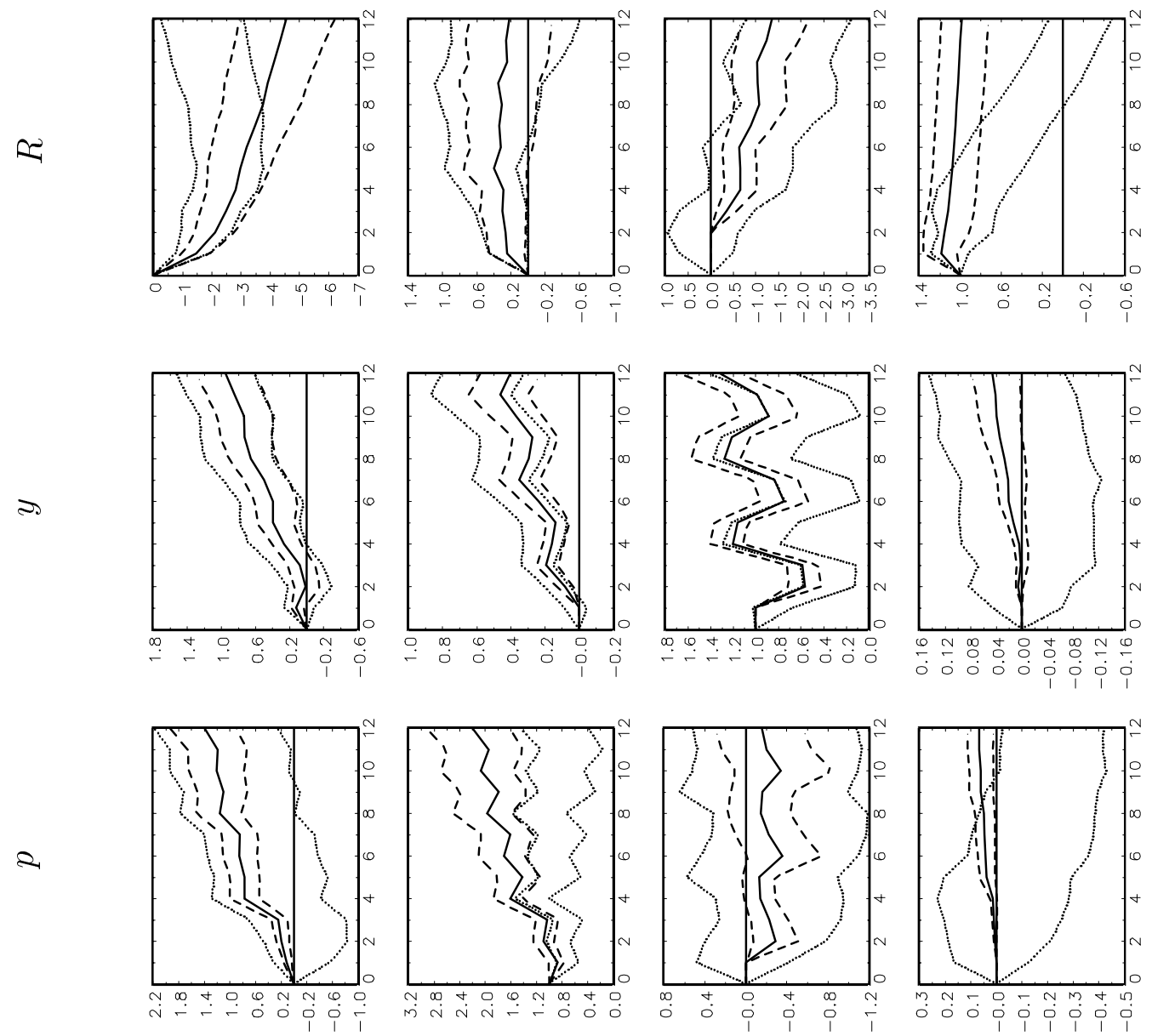

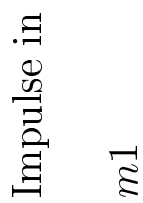
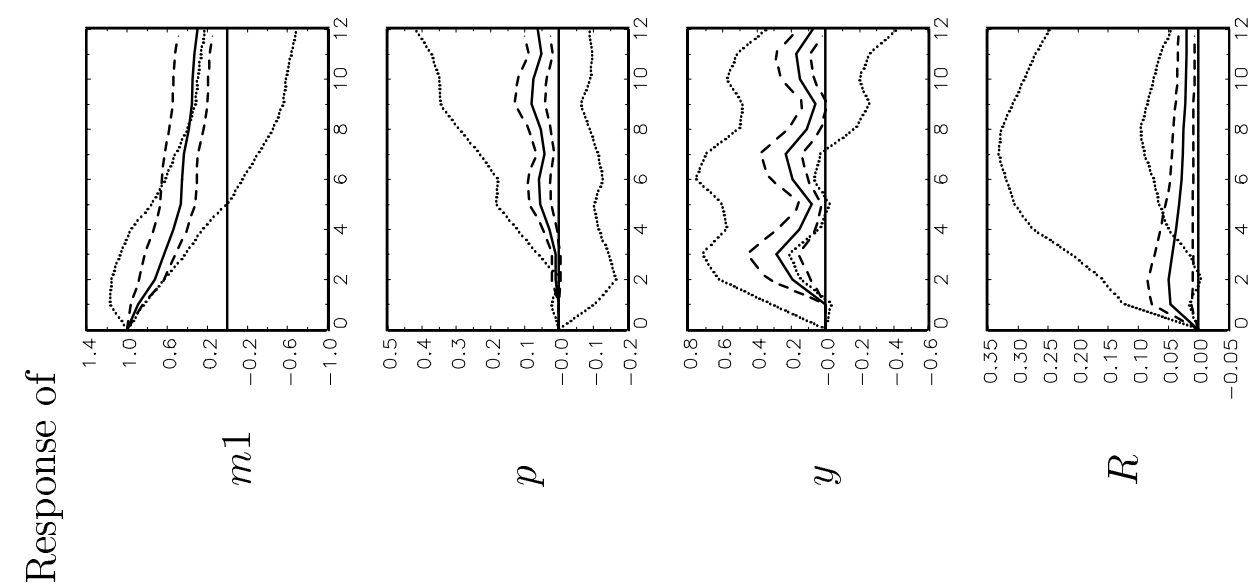

2

s

$\sim$

Figure 2: Estimated impulse responses of restricted VECM for M1 (solid line) with 95\% $C I_{H}$ (dashed lines) and $95 \% C I_{H}$ from fully unrestricted VAR system (dotted lines) 

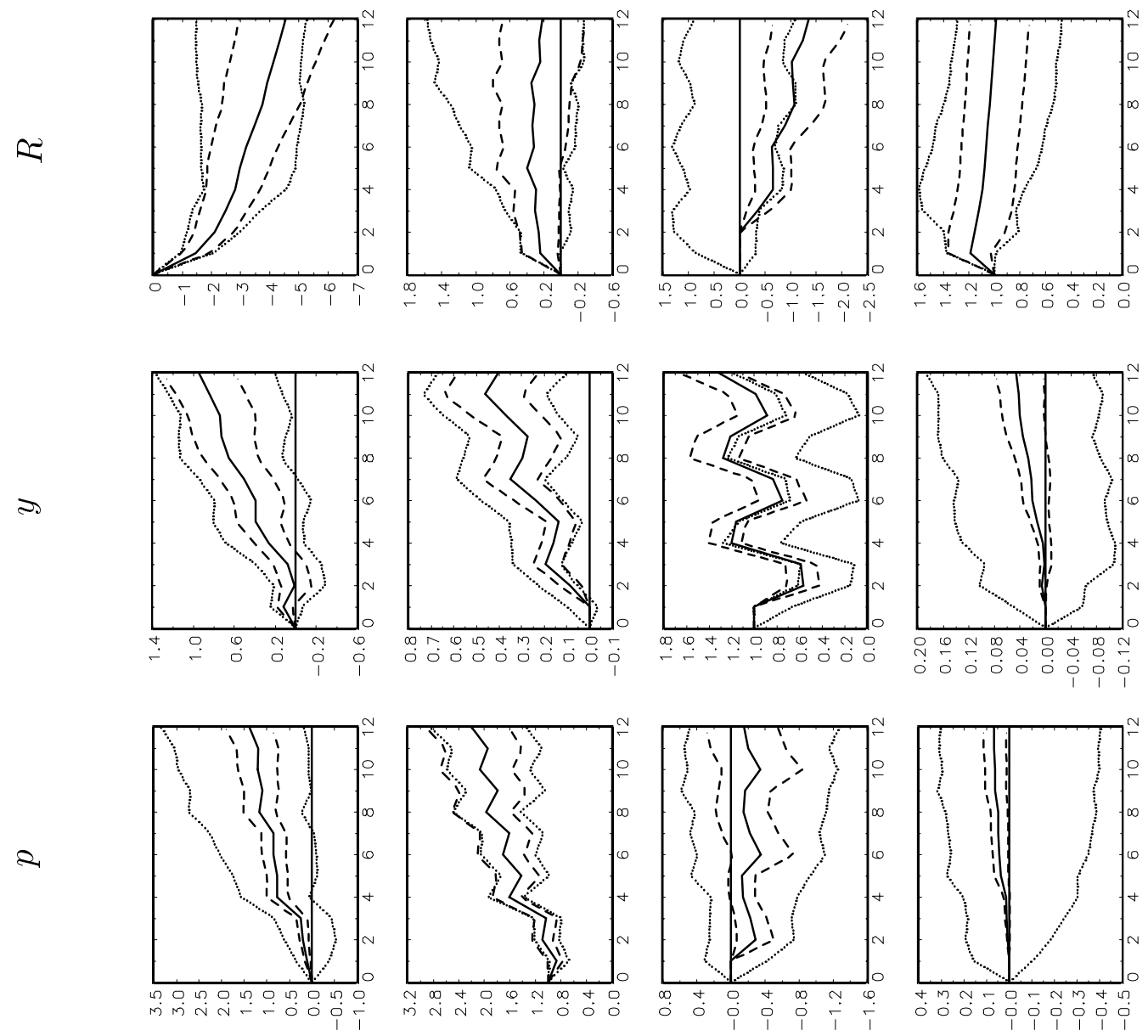

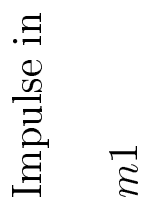
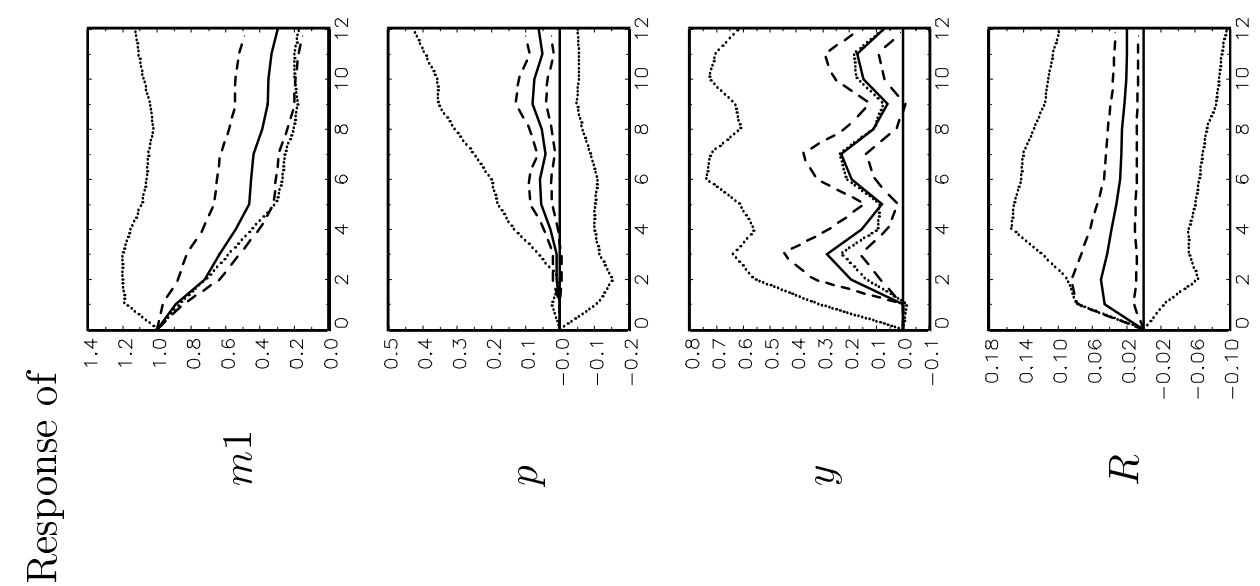

2

S

$\sim$

Figure 3: Estimated impulse responses of restricted VECM for M1 (solid line) with 95\% $C I_{H}$ (dashed lines) and $95 \% C I_{H}$ from unrestricted VECM (dotted lines) 

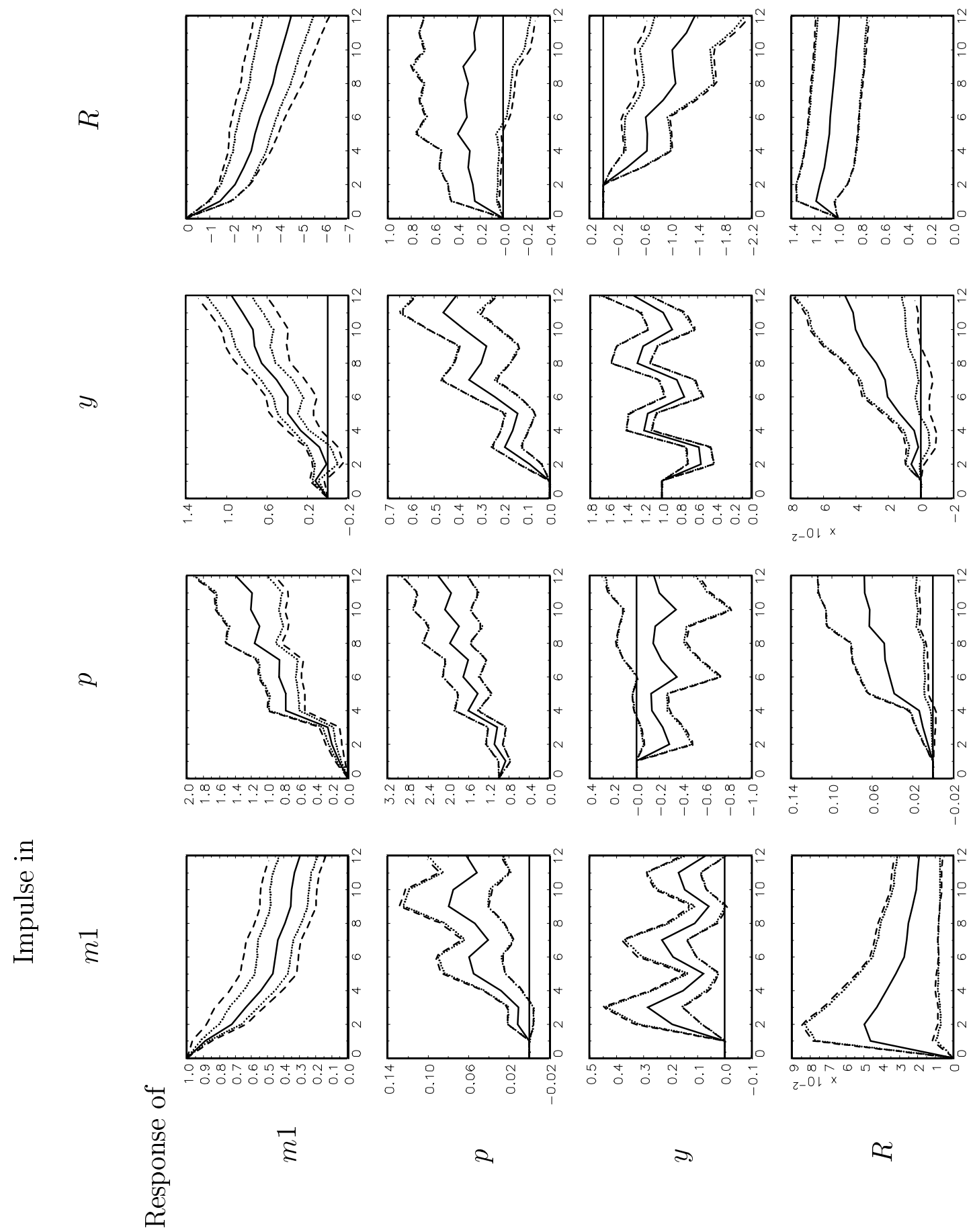

$\rightarrow$

$\sim$

Figure 4: Estimated impulse responses of restricted VECM for M1 (solid line) with 95\% $C I_{H}$ when the cointegration vector is reestimated (dashed lines) or fixed (dotted lines) in every bootstrap drawing 

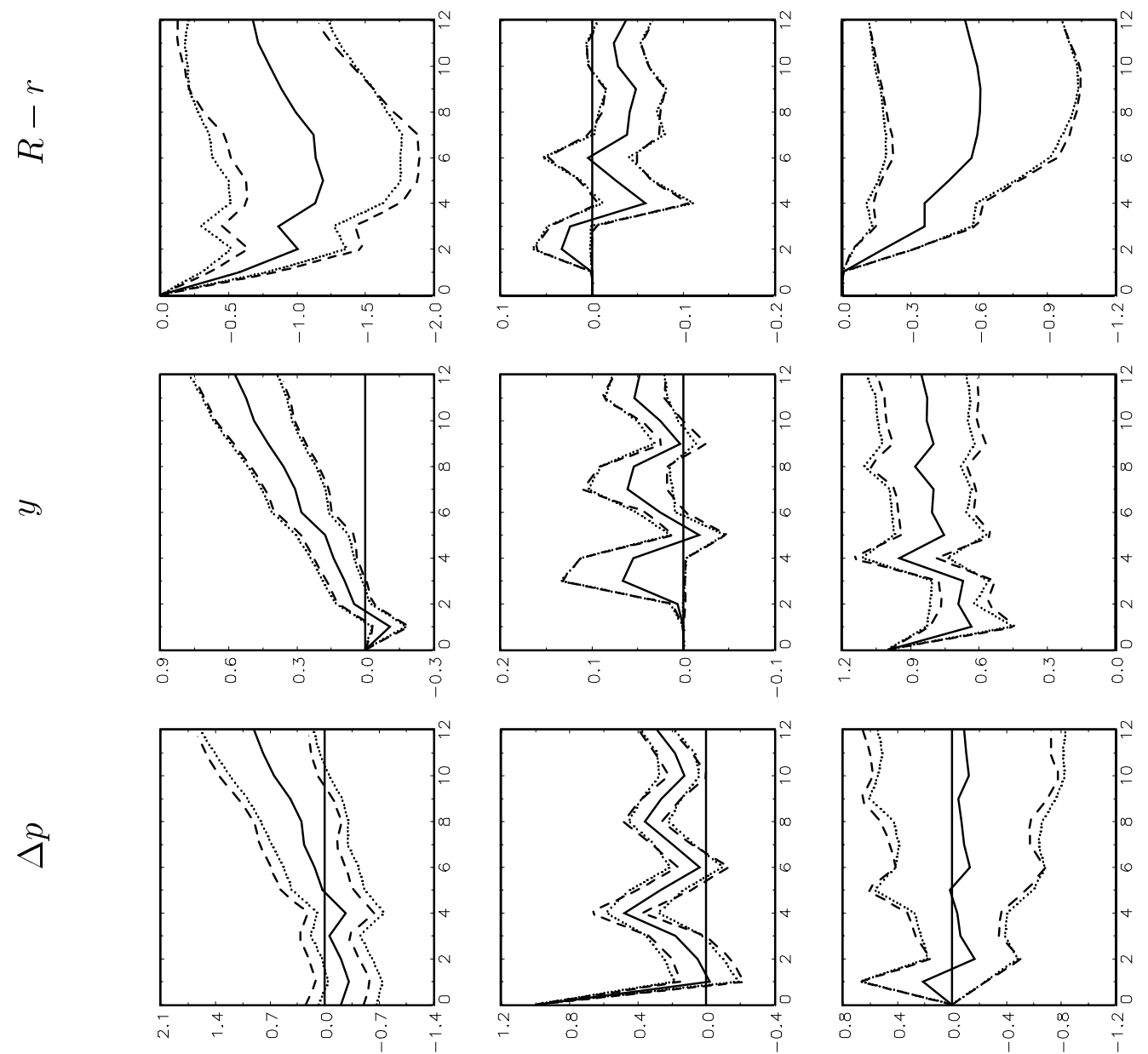

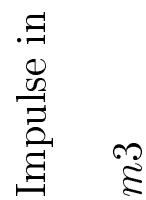
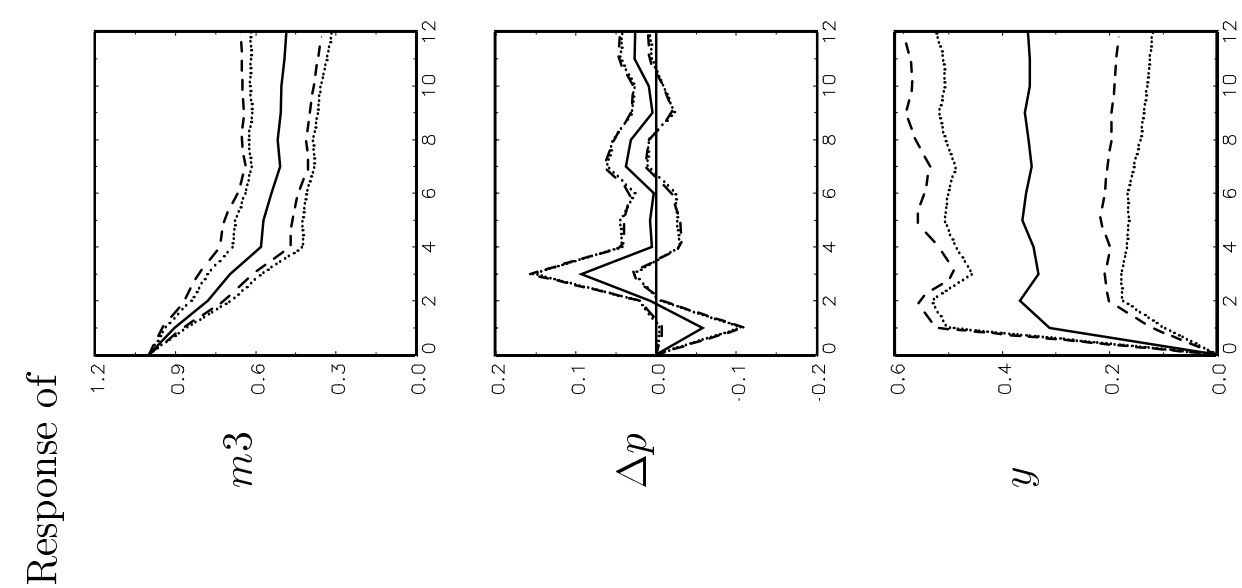

Figure 5: Estimated impulse responses of restricted VECM for M3 (solid line) with 95\% CIs: $C I_{S}$ (dotted lines), $C I_{H}$ (dashed lines) 